\title{
Quantum-like modelling in game theory: Quo Vadis? A brief review
}

\author{
Sudip Patra
}

Center for Complexity Economics, Applied Spirituality and Public Policy (CEASP), OP Jindal Global University, Sonipat, India, and

\author{
Partha Ghose \\ Tagore Center for Natural Sciences and Philosophy, Rabindra Tirtha, India
}

\begin{abstract}
Purpose - The current paper is a brief review of the emerging field of quantum-like modelling in game theory. This paper aims to explore several quantum games, which are superior compared to their classical counterparts, which means either they give rise to superior Nash equilibria or they make the game fairer. For example, quantum Prisoners Dilemma generates Pareto superior outcomes as compared to defection outcome in the famous classical case. Again, a quantum-like version of cards game can make the game fairer, increasing the chance of winning of players who are disadvantaged in the classical case. This paper explores all the virtues of simple quantum games, also highlighting some findings of the authors as regards Prisoners Dilemma game.
\end{abstract}

Design/methodology/approach - As this is a general review paper, the authors have not demonstrated any specific mathematical method, rather explored the well-known quantum probability framework, used for designing quantum games. They have a short appendix which explores basic structure of Hilbert space representation of human decision-making.

Findings - Along with the review of the extant literature, the authors have also highlighted some new findings for quantum Prisoners Dilemma game. Specifically, they have shown in the earlier studies (which are referred to here) that a pure quantum entanglement set up is not needed for designing better games, even a weaker condition, which is classical entanglement is sufficient for producing Pareto improved outcomes.

Research limitations/implications - Theoretical research, with findings and implications for future game designs, it has been argued that it is not always needed to have true quantum entanglement for superior Nash Equilibria.

Originality/value - The main purpose here is to raise awareness mainly in the social science community about the possible applications of quantum-like game theory paradigm. The findings related to Prisoners Dilemma game are, however, original.

Keywords Entanglement, Classicaloptics, Pareto superior outcomes, Quantum-Like Game, Formula for Total Probability5BFTP5D, Prisoners Dilemma 5BPD5D, Nash Equilibrium 5BNE5D, Quantum Probability 5BQP5D

Paper type General review

\section{Introduction}

Modern form of game theory can be rooted back to the seminal works by Neumann and Morgenstern (Bruza et al., 2015), which were based on the neoclassical rationality assumption

(C) Sudip Patra and Partha Ghose. Published in Asian Journal of Economics and Banking. Published by Emerald Publishing Limited. This article is published under the Creative Commons Attribution (CC BY 4.0) licence. Anyone may reproduce, distribute, translate and create derivative works of this article (for both commercial and non-commercial purposes), subject to full attribution to the original publication and authors. The full terms of this licence maybe seen at $\mathrm{http}: / /$ creativecommons.org/licences/by/4.0/legalcode

JEL classification - B23, C00, C02, C60, C70, C71, D80

Received 24 August 2020 Revised 30 August 2020 1 September 2020 Accepted 1 September 2020 
AJEB

4,3

of agents, specifically selfish utility maximization, and also common knowledge of rationality (Common knowledge theory and its outcomes have been modified recently by Khrennikov (Luders, 1951), using rather the quantum frame work of updating beliefs, significantly extending the seminal "agreeing to disagree" model proposed famously by Aumann).

Game theory has then created its own huge literature (Basar et al., 1999; Du et al. ,2001; Fine, 1982; Shafir and Tversky, 1992; Benjamin and Hayden, 2001; Yearsley, 2017), and has now become the currency in which not only standard Economics or Finance is written, but also may other subjects like considerable amount of evolutionary Biology is written. However, since the standard or neo-classical game theory, has been embedded in the rationality framework, real life deviations has been at least non-trivial to depict in that framework. Later there has been a very strong and diverse literature of behavioural game theory, where the utility framework was significantly modified, for example in seminal "inequity aversion" games by Fehr and Schmidt.

Hence we accept that behavioural Economics or Finance studies have modified the classical game theory, mainly by modifying the simple selfish utility maximization criterion to which players should respond, for example in the inequity aversion game players are constrained to respond by the differences between their and other players' payoffs, which also generates disutility among players if the payoff gaps increases, which might be near to altruistic behaviour, in case of disutility generated to one player due to ones payoff increasing compared to the other. However, we just mention here that the dense literature of the extant behavioural game theory is not fully based on a single coherent and comprehensive probability theory framework, and it is not surprising since heuristics-based set ups were adapted to avoid typical probabilistic decision-making framework. Hence, while turning to quantum-like modelling in game theory we aim for providing a comprehensive probabilistic structure which might be able to resolve different "anomalies" or deviations observed from the predictions of classical Nash Equilibria (NE here). NE or Nash equilibrium, a central concept of game theory, is a stable equilibrium position where every player is playing ones best strategy given every one else is player their best strategies, such that it is of no utility of a player to deviate from the position unilaterally.

$W e$ would also like to emphasize that classical or traditional game theory literature is vast and versatile. Though the concepts of selfish utility maximization, or full rationality, or complete information were introduced in the first phases of the literature, soon many modifications happened (Yearsley, 2017) where modified utility set ups (mainly in behavioural game theory literature), games under incomplete information and uncertainty were studied. We always remind ourselves that game theory as a literature is evolving very rapidly. Particularly, since the seminal experimental observations by cognitive scientists (Krueger et al., 2012), who prescribed different heuristics based explanation of human choice behaviours under uncertainty, behavioural game theory has rapidly adapted such findings.

Since late 1990s (Busemeyer and Bruza, 2012; Iqbal and Abbott, 2009; Iqbal and Abbott, 2010), quantum-like modelling in social science has emerged as a true alternative in understanding real agent's behaviour. However, we always remind readers that there is no real quantum physical process being claimed as underlying the human behaviour, or market behaviours. We focus on the novel mathematical and logical concepts, tools or frameworks widely used in quantum mechanics, or quantum field theory, or even in classical optics (Haven and Khrennikova, 2018), which may be extended to decision theory or cognition in general. 
However, contribution of quantum-like modelling (for a comprehensive introduction we can refer to Haven and Khrennikov (Iqbal and Abbott, 2009) in social sciences, and game theory particularly is more fundamental. Quantum-like framework allows for a comprehensive framework where different deviations from neoclassical decision theory can be depicted consistently and coherently, for example, order effects in decisionmaking, failure of sure thing principle in decision-making under uncertainty, contextuality in decision-making, and alike. Again, this also means that the comprehensive framework would also contain the standard results when appropriate,

Quantum-like modelling in game theory for example in Khrennikov's (Luders, 1951) extension of common knowledge theory we get back the neoclassical standard results when condition of full information for rational agents prevails.

In the current paper, it would not be possible to survey the huge literature, rather we attempt to straight away turn to 'quantum like' game theory, and then present our own framework. We may remark that quantum-like game theory paradigm can be perceived from multiple directions, one (Iqbal and Abbott, 2009) in which real people play games in standard set up, for example prisoner's dilemma set up [PD henceforth], and then conditional probabilities of choosing moves are computed from real data, if deviations are observed from typical dominant Nash Equilibrium predictions, then such deviations are explained via quantum-like framework. The other direction being designing better and more efficient games, such that even classical rational players may use such novel features, or resources, to achieve superior outcomes which are not possible in standard game formats. Inter or intra system entanglement is one such feature which has been shown to be used to achieve superior outcomes (Li et al., 2006).

The organization of the paper is the following: literature review section briefly explores aforementioned directions, a brief section on examples of quantum games, the following section is a proposed framework by the authors which has recently been developed by us, and hence the discussions would mostly be around one exemplary famous game, the prisoner's dilemma game, PD in short. Then the paper discusses some potential future research and presents conclusion.

\section{Literature review}

First, let us see some crucial differences between sociological or behavioural approaches to game theory and quantum-like approach. We refer to the famous PD game for this purpose, in the simplest formulation of the game with two players Alice and Bob, and two possible moves for each player $\mathrm{C}$ (cooperation) and $\mathrm{D}$ (defection), we get a standard payoff matrix, where if players cooperate the payoff is greater to each than if both defect, but again if one defect sand other cooperates the defector would get a very high payoff and the co-operator a very low payoff as compared to other scenarios. In this game if we start with the standard utility framework, and common knowledge of selfish rationality, then it is well known that players would be forced to choose [D, D], which is an inferior outcome, even though this is the dominant NE.

However, as sociologists and behavioural economists have noted since long (Haven and Khrennikova, 2018) real players (often called as "naïve" players!) do play or choose strategies differently, many players do choose $\mathrm{C}$ instead of $\mathrm{D}$, hence the question arises why? What might be the underlying social or psychological processes responsible for such deviated outcomes? In a widely known study, KDF (based on authors names as in the reference section) (Bruza et al., 2015; Myerson, 2013) proposed "social projection hypothesis", the study explored various moral theory perspectives to account for such deviations: reciprocity approach, benevolence approach, team 
work approach which all indicate that players would expect other players also to behave in their way of just and fairness, then there might be error approach also which holds that $\mathrm{D}$ being the correct move for both players, naïve players do make wrong moves at times. However, any specific theory would not explain real moves under all scenarios.

Now coming back to quantum-like game theory, we observe that here the purpose is related but fundamentally, from the perspective of probability framework, i.e. how the real moves chosen by real players, or the results of real games might be described comprehensively based on a single coherent probability or logical framework. Certainly, such a description would not be possible in standard Boolean logic or measure theory framework, which is the underlying mathematical theory for neoclassical game theory, PD being one example.

Shafir and Tversky (Benjamin and Hayden, 2001) initiated in some way the behavioural game literature when they studied the experimental results of real PD games, it was keeping upto standard predictions when in some games a player was informed about the move of the other, so when Alice knew that Bob's move was D she chose $\mathrm{D}$ with a high probability and also chose $\mathrm{D}$ with a nearly high probability when she Knew Bob would choose C, however when the players knew nothing or were uncertain about other players' move then probability of $\mathrm{C}$ went up significantly. This iconic result has been repeated numerous times (Iqbal and Abbott, 2009; Tversky and Kahneman, 1973), which clearly challenges the standard game theory predictions.

The deeper question is then, whether human cognition mainly under "uncertainty" can be at all described by classical probability theory, hence standard game theory? Certainly there are many studies (Busemeyer and Bruza, 2012; Bruza et al., 2015) which do suggest that $\mathrm{CP}$ [1] theory might be very critical for accounting quite a few human cognitive behavior, for example, Dutch Book game (Busemeyer and Bruza, 2012; Bruza et al., 2015). However in general it might be possible to generalize the structure more, which has been provided by QP modelling, quite comprehensively (for general references (Haven and Khrennikov, 2013; Myerson, 2013)).

The central issue is the so-called FTP or formula for total probability, based on CP theory axioms probability for any event $\mathrm{A}$ to happen conditioned on $\mathrm{X}$ happening or not is provided by a simple expression: $\mathrm{P}(\mathrm{A})=\mathrm{P}(\mathrm{X}) \mathrm{P}(\mathrm{A} \mid \mathrm{X})+\mathrm{P}\left(\mathrm{X}^{\prime}\right) \mathrm{P}\left(\mathrm{A} \mid \mathrm{X}^{\prime}\right)$, where $\mathrm{X}^{\prime}$ is the negation of the event $\mathrm{X}$. Hence if we apply this FTP for PD game then we obtain $\mathrm{P}$ $($ Dplayer1 $)=\mathrm{P}($ Dplayer2) $\mathrm{P}($ Dplayer1|Dplayer2 $)+\mathrm{P}($ Cplayer2 $) \mathrm{P}($ Dplayer1 $\mid$ Cplayer2 $)$. However, Tversky and Shafir experimental outcomes could not be explained by the same formulation. FTP is certainly a general formula.

QP theory (we briefly explore the basic tenets of QP theory in the Appendix), in general allows for extra perturbative terms in the FTP, which are known as to be the "interference" terms. The basic math of computing probabilities in QP framework is provided by the famous 'square of the amplitude' rule or Born's rule, which when applied to FTP straight away generates 'interference' terms. Such interference terms have their own history with famous double slit experiments with photons or electrons (Haven and Khrennikov, 2013).

Here the usage of such modified FTP can generate probabilistic results which are near perfect match with experimental data, for example in real PD games under uncertainty condition. Hence, in the next subsection we briefly survey such models based on QP theory. 


\subsection{Two directions or approaches}

2.1.1 Describing and updating belief states/cognitive states of players, and computing "interference terms". In the Appendix, we provide a demonstration or derivation of interference terms in the modified FTP, when QP framework is adapted for decision-making, i.e. computing probabilities of variables assuming certain values (in this case bivariate variables, which can be generalized), and updating the 'cognitive' or belief states of agents. Here we would also like to mention that in QM there are different formalisms of updating states; Neumann orthogonal projection where purity of states are not conserved (so called wave function collapse and hence 'measurement' problem has resulted in the history of quantum foundations, with continual debate), Luder's update rule where pure states updates as pure states (there have been many recent studies on distinguishing between Neumann and Luder ansatzes (Shafir and Tversky, 1992), and then POVM or positive operator based measurements and updating which are very relevant for cognition modelling (Yearsley, 2017); we provide a brief mathematical detour in the Appendix.

Khrennikov and Haven (Iqbal and Abbott, 2009) have provided detailed computations of quantum FTP, based on results obtained from simple real games, PD included. One important inference is that interference effects shows up under the context of uncertainty, which is again supported by the findings of authors as mentioned above. In classical game theory there is not much fundamental difference between risky and uncertain contexts, hence decision-making under uncertainty context seems to be a fundamental contribution by quantum cognition modelling. Another important work done by Khrennikov (Myerson, 2013) and Haven and Khrennikova (Kahneman et al., 1982) is construction of mental or cognitive states from the computations of phases in quantum FTP.

\subsection{Designing games with superior equilibria}

The quantum game theory literature has another very significant side, which is designing superior games, FTP certainly plays an important role there too, but the main focus is not just to analyse real players moves but to equip real players with such resources (for example pure or classical entanglement) which would help them achieve superior strategy profiles and hence Pare improved equilibria. Here again we are not assuming anything further than selfish utility maximization.

Designing of quantum games has a long history (Fine, 1982; Khrennikov, 2015; Krueger et al., 2012), the main technique being to make players share a physical system in such a way that the payoffs of each player now not only depends on the 'classical' moves of the players but also on the nature of physical systems player share.

Certainly, then EPR type physical systems can be shared by players with entanglement as the central feature. Works (Fine, 1982; p. 27) show that entanglement guarantees superior equilibrium outcomes. However there are alternative thoughts too in the quantum game literature, for example in ref (Khrennikov, 2015; Krueger et al., 2012) we find a quantum prisoners dilemma game based on the violations of Bell inequalities, in form of Fine's inequalities (Haven and Khrennikova, 2018) actually does not give rise to superior NE, rather $\mathrm{DD}$ in a $2 \times 2$ game still remains the NE4.

However, the same papers (Khrennikov, 2015; Krueger et al., 2012) also show that game designed based on maximal CHSH [2] violation for Matching Penny game generates superior $\mathrm{NE}$, so there is still some work to be done to find a general theorem or condition for establishing superior $\mathrm{NE}$ with $\mathrm{QP}$ framework. Is pure quantum entanglement a necessary condition for designing superior than classical games? And it is in this backdrop we, current authors, present briefly our own framework. 
AJEB

4,3

\subsection{How quantum games are made?}

As described in the literature review quantum game design is a promising and evolving literature where at least one player in the game has access to quantum strategy. As has been mentioned earlier to have certain quantum-like features in the game, players at times are required to share among themselves actual quantum systems such that true quantum entanglement can be present. It has been shown that presence of entanglement in the shared physical systems used by the players generates superior games with improved equilibriums.

However, more simply, we can think not all players having access to quantum strategies, where such strategies are abstractly represented by GATES [3].

As used profusely in classical information theory, in quantum computation also we can think of different unitary matrix representation of GATES. Unitary operators are such that, its adjoint is equal to its inverse, hence $\mathrm{U} \mathrm{U}^{*}=\mathrm{U}^{*} \mathrm{U}=\mathrm{I}$ where $*$ symbolizes the operation of transposed complex conjugation. $\mathrm{I}=\left(\begin{array}{ll}1 & 0 \\ 0 & 1\end{array}\right)$ is the identity matrix. $\mathrm{U}$ is any general unitary matrix. Hadamard GATE is the unitary matrix represented in $2 \times 2$ form as $\mathrm{H}=\frac{1}{\sqrt{2}}\left(\begin{array}{cc}1 & 1 \\ 1 & -1\end{array}\right)$

Hence if $\mathrm{H}$ operates on $\mid 0>$ or $\mid 1>$ states we get resultant superposition states:

$$
\begin{aligned}
& \left.\mathrm{H}\left|0>=\frac{1}{\sqrt{2}}(|0\rangle+\mid 1>) \text { or }\right|+\right\rangle \text { state } \\
& \left.\mathrm{H}\left|0>=\frac{1}{\sqrt{2}}(|0\rangle-\mid 1>) \text { or }\right|-\right\rangle \text { state. }
\end{aligned}
$$

where as usual $\mid 0>=\left(\begin{array}{l}1 \\ 0\end{array}\right)$ and $\mid 1>=\left(\begin{array}{l}0 \\ 1\end{array}\right)$ the computational basis.

Hence, in a simple Penny Flipping game, the rule is that Alice first has the opportunity to operate her strategy on the state of the game, i.e. on the penny (not disclosed), and then Bob, and then Alice and so on. Also imagine that Alice is the player having access to the Hadamard GATE operation on the penny, then it can be shown whether Bob plays pure strategies (either flip the penny or not) or a mixed strategy (of say 0.5 probability of flipping and 0.5 of not flipping), it is Alice who would always win the game. Hence, we can theorize if one of the players has access to such quantum strategies then his or her expectation of payoffs is at least as great as in the case of pure classical game.

Using such quantum game designs in practice however, is still an area open to research and interpretations. Specifically use of pure quantum entanglements in the design of games is very hard, since atmospheric decoherence might easily disturb such a delicate setting. Here comes the novelty of some preliminary results found by the authors of this review, which we turn to now.

\section{Examples of quantum games}

\subsection{Sequential moves game: Penny flipping}

In the simple classical version of the game, say Alice and Bob are the players (rational utility maximizers). The rules of the game can be Alice first decides to Flip (F) or not Flip (N) the penny, next move is for Bob, he can choose $\mathrm{N}$ or $\mathrm{F}$, and then again for Alice between $\mathrm{N}$ or $\mathrm{F}$. 
Additionally Alice wins if the final state of the penny is heads up, and Bob wins if final state of the game is tails up. No player is allowed to see the intermediate state of the game.

It is easy to show that if the initial state of the penny is heads up, then the below strategic form can be generated: (say the initial state of the penny was heads up) (Table 1).

Now to "quantize" the game, we need to think that the game's state can be described in terms of Qbits $(\mid 0>$ or $\mid 1>)$, and the strategies which players use can be represented by Unitary operators in general/GATES like Hadamard GATE for a quantum strategy or I or X operators for classical strategies(I representing $\mathrm{N}$, which means that the player has not

Quantum-like modelling in game theory

flipped the coin, since the state of the coin say $\mid 0>$ remains the same after this operation, I is nothing but the identity matrix $\left[\begin{array}{ll}1 & 0 \\ 0 & 1\end{array}\right]$ and $\mathrm{X}$ representing $\mathrm{F}$, which means the player has flipped which changes the state of the penny, $\mathrm{F}$ is represented by the X Pauli Matrix, $\left.\left[\begin{array}{ll}0 & 1 \\ 1 & 0\end{array}\right]\right)$

There are some observations which follows:

- If Alice plays a Hadamard GATE strategy, then the immediate state of the penny after the move would be a superposition state: say the initial state of the game was $\mid 0>$, then $\mathrm{H}\left|0>\frac{1}{\sqrt{2}}(|0\rangle+\mid 1>\right.$ )or $\mid+>$

Then if Bob only plays classical strategies (I or X), then Alice always win if the initial state of the penny was $\mid 0>$. As when it finally comes to Alice she can choose to Flip again, and the state would always get back to $\mid 0>$ or heads up. Symbolically: $\mathrm{HIH}=\mathrm{HXH}=\mid 0>$.

It can also be shown that if even Bob plays a mixed classical strategy with some mix of I and X (in this case with 0.5 probability each), Alice would always win in this scenario.

- If, however, both Alice and Bob have access to pure quantum strategies, i.e. say they both play Hadamard GATE only, then there is no scope for a Nash Equilibrium to emerge.

- However, if both players play mixed quantum strategies, then there is a NE guaranteed.

\section{Cards Game:}

This game has different nature altogether, from the above type game, since "quantizing" a classical card game actually makes the game fairer. For example, if Bob had 2/3 probability of losing the classical game of cards against Alice, if Bob get access to a quantum "query" machine he can have fair odds of winning the game. Hence, quantum game design can also be used for making an unfair classical game fairer.

In a simple version of the classical card game, say Alice has 3 cards, with 2 cards with same signs on back and front, and 1 card with different signs on the back and

\begin{tabular}{lcrr}
\hline & BOBN & F & \\
\hline ALICE NN & $(1,-1)$ & $(-1,1)$ & Table 1. \\
NF & $(-1,1)$ & $(1,-1)$ & $(1,-1)$ \\
FN & $(-1,1)$ & $(-1,1)$ & Quantum vs classical \\
FF & $(1,-1)$ & game \\
\hline
\end{tabular}


AJEB

4,3

front of it. The rule of the game is that Alice puts all the cards in a black box and shakes the box randomly, such that the cards get arranged in a random fashion. Then Bob is asked to pull a card, without flipping it. Now if the players find that the card being pulled has same sign on both sides, Alice wins with a payoff +1 , and if the card has different signs on both sides Bob wins with a payoff +1 , the looser gets a payoff -1 in all cases.

It is simple to see that this game is unfair, since expected payoffs of Alice and Bobs are: for Alice $2 / 3(+1)+1 / 3(-1)=1 / 3$, and for Bob $2 / 3(-1)+1 / 3(+1)=-1 / 3$, or in other words Alice has a probability of $2 / 3$ to win, and Bob has a probability of $1 / 3$ to win. For attracting Bob into the game, Alice also gives Bob one chance to raise a query about the cards in the black box, after getting the answer he may decide to play or leave. However, in this case also the game remains unfair, since Bob gets information about one card only.

\subsection{The quantized game case}

Let us assume 0 as one sign or label on the card and 1 being another sign, hence when the cards are in the Black box with their top up positions, then the general state of the cards can be described by a Qbit $=|r\rangle=\mid r_{0} r_{1} r_{2}>$ with $r_{I}=0$ or 1 . Now Bob would like to gain information about the cards by using a 'quantum' query machine. Again we assume that the machine state depends on $|r\rangle$, generally such a machine can be described as an Unitary matrix $\mathrm{U}=\left[\begin{array}{cc}1 & 0 \\ 0 & e^{i \pi r_{i}}\end{array}\right]$ ence if $\mathrm{i}=0$, we have $\mathrm{U}=\mathrm{I}$ and if it is 1 we have $\mathrm{U}=\mathrm{Z}$ (Z GATE, $\left[\begin{array}{cc}1 & 0 \\ 0 & -1\end{array}\right]$ ), now since Bob has also access to quantum strategies, namely, the afore mentioned $\mathrm{H}$, he sandwiches $\mathrm{U}$ between two Hs, to get the following state:

$$
\mathrm{HUH}=\left[\begin{array}{cc}
1+e^{i \pi r_{i}} & 1-e^{i \pi r_{i}} \\
1-e^{i \pi r_{i}} & 1+e^{i \pi r_{i}}
\end{array}\right] \text {, this becomes the query machine for Bob, where he can }
$$

input a Qbit state like $\mid 000>$ and get an information $\mid r>$, or in other words if Bob inputs $(\mathrm{HUH} \otimes \mathrm{HUH} \otimes \mathrm{HUH})|000>=| \mathrm{r}_{0} \mathrm{r}_{1} r_{2}>$, or in other words Bob gets information regarding the top side of all the cards at once!

Hence Bob knows that the state of the up sides of whole system of cards can either be:

$$
\mathrm{S}_{0}=\{|0>,| 0>, \mid 1>\} \text { or } \mathrm{S}_{1}=\{|1>,| 1>, \mid 0>\}
$$

With this information Bob knows if the state in the Box is $S_{0}$ and he pulls a card with $\mid 0>$ as the upside then he and Alice have equal chance of winning, and thus continues the game. But if he pulls a card with $\mid 1>$ side up, then he knows he has pulled a losing card and should quit the game. The opposite holds if the state of the upside cards in the Box is $\mathrm{S}_{1}$. Hence, this game is not securing win for Bob, but making an unfair game fairer.

\section{4. "Classical optical" modelling of PD game: a brief discussion}

Recently (Iqbal and Abbott, 2009) good amount of attention has been directed toward studying classical entanglement phenomenon, exhibited by classical optics or even by classical mechanical systems. The critical difference lies in the fact that true quantum entanglement or non-local correlations (without violating relativistic causality for sure) can be exhibited either by space- like separated subsystems or entanglement or coupling between degrees of freedom of the same system (for example path-spin entanglement for 
electrons, etc.), whereas classical entanglement is only for multiple degrees of freedom for a single classical system.

Hence based on the above discussed literature of designing superior strategies or games relative to standard classical games, the question arises whether the shared physical system among the players might have 'classical entanglement' and still generate superior strategies which results into superior NE?

Earlier literature (Li et al., 2006) have suggested that quantum entanglement guarantees better NE generation, but now we have demonstrated even much simpler shared physical systems might generate similar NE, for example for PD game. For the fuller mathematical treatment, we refer to our recent work (Li et al., 2006). Here we briefly mention the salient features of our framework.

To re-design the PD game such that superior strategy profiles emerge, we closely follow the ref (Rasmusen, 1994) with (a) sources of two polarized classical light beams each for one player (b) a set of optical instruments which help each player to manipulate each light beam in a strategic manner (c) a measurement device (in this case polarization analyzer) which generates payoffs for every player

${ }^{4}$ In this related studies, classicality of the shared physical system between the players has some degree of correspondence to factorizability of joint probabilities, however nonfactorizability of those joint probabilities may not always mean violations of Bell inequalities, hence non-factorizability is a sufficient but not necessary condition for "quantumness" of the shared physical system based on the polarization sates of each optical beam. Again, the game set up is a common knowledge to all players, say to Alice and Bob in case of a $2 \times 2$ game.

Here then, we assign two vectors $\mid \mathrm{C}>$ and $\mid \mathrm{D}>$ to two possible classical strategies $\mathrm{C}$ and $\mathrm{D}$ (co-operate and defect namely) which spans the $2 \mathrm{D}$ Hilbert space $\mathrm{H}$ of a polarized beam. Hence state of the game at every instant is provided by a vector in the tensor product space $\mathrm{HA} \otimes \mathrm{HB}$ spanned by the basis $|\mathrm{CA}>\otimes| \mathrm{CB}>,|\mathrm{CA}>\otimes| \mathrm{DB}>,|\mathrm{DA}>\otimes| \mathrm{DB}>,|\mathrm{DA}>\otimes| \mathrm{CB}>$, now if $\mid \mathrm{C}>$ is assigned as $\mid 0>$ and $\mid \mathrm{D}>$ is assigned as $\mid 1>$, two standard basis vectors on the Poincare sphere [4], representing horizontal and vertical polarization respectively, then, the 'optical' states with Alice and Bob will be: $|\psi>\mathrm{I}=\cos \Theta \mathrm{i}| \mathrm{C}>\mathrm{I}+\mathrm{e}$ i $\varphi \mathrm{i} \sin \Theta \mathrm{i} \mid \mathrm{D}>\mathrm{I}$ where I denotes Alice or Bob. With $2 \Theta$ in the range $[-\pi / 2,+\pi / 2]$, and $2 \varphi$ in the range $[0,2 \pi]$.

Based on this representation we can then define, 'strategy space' of the players using Unit Matrices [5] $U$ (2), where $U$ is the unit matrix symbol, with the property that $\mathrm{U}^{*} \mathrm{U}=\mathrm{UU}^{*}=\mathrm{I}$, where $*$ here means the operation of complex conjugate transposition, and I is the identity matrix. Hence, we can define $\mathrm{U}(0,0)$ which is the identity matrix or operator in operator representation, and the other matrices in form of Puli Matrices. Again based on the algebra followed by the Pauli matrices $[\sigma \mathrm{i}, \sigma \mathrm{j}]=2 \mathrm{i} \epsilon \mathrm{ijk} \sigma \mathrm{k}$, which forms a closed set of nontrivial operators, one can associate at most three independent strategies. Another important advantage of this modelling is since the results are based on general Non-Abelian algebra, there is no presence of Plank's constant in our equations, which has always been a worry in quantum-like modelling, due to lack of proper interpretation.

Hence, we find three independent strategies emerging out, $\mathrm{C}$ which corresponds to $\sigma_{3}, \mathrm{D}$ which corresponds to $\mathrm{i} \sigma_{2}$ and $\mathrm{L}$ (say, which means abstain) which corresponds to $\sigma_{1}$. Hence, we already see extended strategy profiles for players, it may also be noted that a linear combination of $\mathrm{C}$ and $\mathrm{L}$ would produce $\mathrm{H}$, the well-known Hadamard Gate.

We would request the readers to refer to our detailed framework (Li et al., 2006) for the full mathematical model. Here we just mention that based on the extended strategy profile, if classical entanglement feature is introduced in the system of optical beams, then D,D no 
- We have distinguished our frame work of designing strategic decision-making in form of a prisoners dilemma game (PD), which is based on novel features of classical optics; mainly classical entanglement which is robust; from pure 'quantum like' description of similar games, which are often called as 'quantum prisoners dilemma' (QPD).

- We have been able to demonstrate the main point of departure from neoclassical game theory, that is, providing a mechanism to bypass the stubbornly dominant 'defection' Nash equilibrium in a PD.

- We have demonstrated that based on classical optical modelling (COM, a frame work of cognition model which we have developed recently) or designing of PD we can achieve, or demonstrate Pareto superior equilibrium outcomes even in nonrepetitive PD.

- Our design of PD is thus an alternative to extant models which try use pure quantum entanglement (which is very fragile due to noise in the environment, in this case information environment), and hence our design is practicable while using simple optical tools, namely polarization. We have provided a detailed description of our design in the paper with simple geometric representations of decision moves by players via rotations of Poincare sphere. Such correspondence between decisionmaking and rotation operations on Poincare sphere is an elegant way to capture new strategy profiles of players, and Pareto improved equilibrium points.

Another novel feature of 'quantum like' game theory has been to compute conditional probabilities, and provide an alternative formula for total probability (FTP), which demonstrates departures of behaviour of real players from predicted Nash equilibria. Our FTP is based on the earlier developed COM framework, which relies on Luder's updating rule rather than Neumann projection rule used often in extant QPDs.

Overall, a playable frame work is built in current paper with future progression towards repeated PD and $n$ persons $\mathrm{PD}$.

\section{Further discussions}

The main contributions of quantum-like modelling in game theory have been two fold, one describing real plays under uncertainty more faithfully, where Khrennikov and Haven [op cit] have suggested one mechanism to reconstruct cognitive states of players based on the interference terms computed from the quantum FTP, and two, designing superior games. QPD design is efficient since we obtain Pareto improved equilibria in the single shot game itself, rather than playing the game for many rounds, which is the standard way to have cooperative equilibria emerge in such games. The current authors have demonstrated recently (Li et al., 2006) that rather than pure quantum resource based game design if we use much simpler classical optics based design/ resources then the novel feature of 'classical' entanglement can be exploited to arrive at similar superior equilibria.

One of the main claims of quantum game theory is that of quantum supremacy. In other words if in a standard game (for example penny flipping game, or any such zero sum game, where utility functions are symmetric and opposite to each other's players, such that ones gain is exactly others loss) if one player is endowed with a quantum strategy, and the other 
player has no access to it, then the former would always win irrespective of whether the later plays pure or mixed strategies.

By quantum strategy here we mean analogous operations to Classical GATES like NOT, AND, OR, etc. for example one of the most important GATE or strategy here is Hadamard GATE which can be given a $2 \times 2$ matrix representation, such that when this operation is done on the state of the game being played a non-trivial superposition state is resulted, which is fundamentally different from that in classical games. In case of penny flipping game it can be easily shown that if one player has access to such a strategy (generally any unitary matrix representation but specifically Hadamard GATE) then irrespective of pure or mixed strategies played by the other player, the former is sure to win.

We have also seen, as in the card game, that if one of the players get access to quantum strategies (in this case a quantum query machine) then the information gained will be greater than in the case of classical game, which would make the game fairer for the player.

QP framework can also be used in describing noise or error in games, with the help of Positive operator representation of measures or moves in the game.

Among many striking features of cognitive systems (for example contextuality which we have based our framework on) is 'noise'. There may be many sources of noise (Yearsley, 2017), either based on faulty framing of experiments, here for example, faulty framing of questions/questionnaire posed to agents, or inability of agents to provide sharp responses (yes or no, 0 or 1 etc.). Recently, POVM has been used in cognitive modelling related to describing choice behaviour of agents under uncertainty; this is a very helpful tool in describing agents' behaviour in case of uncertainty in financial markets since many interesting results like order effects can be explained.

Noise in the system has an important interpretation in the decision theory literature; for example, say due to some noise in the final choice action, or due to some error, the agent rather choosing the optimal chooses a wrong option, now such actions can be represented by positive operators, rather than more stringent projection operators as described earlier. One important example could be the suboptimal choice of portfolios by agents, which then can have significant impacts for mispricing of assets.

In real cognitive experiments, or, choice making by agents even under full information, there can be errors, for example if there are two mutually exhaustive choices A and B, then there may be agents who would rather prefer A but instead choose B and vice versa. Hence in such cases choices cannot be represented by typical orthonormal projector operators, rather we would need some operators, say EA whose expectation value in the state $|A\rangle$ is close to 1 but with an error, similar for $E B$. In the basis of $\{|A>| B>$,$\} such operators can be$ termed as positive operators.

Positive operators such as these, are, one, complete: $\mathrm{EA}+\mathrm{EB}=1$, and positive, since their Eigenvalues are $>0$, which also means, for any general state (mixed state like $\rho$ ), Trace $(\rho \mathrm{EA}) \epsilon[0,1]$, and $\sum($ Trace $\rho \mathrm{EA})=1$, where sum is over the chosen basis.

As we have described earlier Positive operators are a class of projection operators which have more general properties, for example, if $\mathrm{E}$ is one positive operator then it can be conceived of as $\mathrm{E}=\mathrm{M}$ 'M, where $\mathrm{M}$ is a self-adjoint operator and $\mathrm{M}$ ' is the transpose conjugate of $M$, such that for all such observations $\sum M^{\prime} M=I$ where I is the identity operator. Again, M can be given a square matrix representation, for example, if $\epsilon$ is the noise in the system then: 
AJEB

4,3

$$
\mathrm{M}=\left[\begin{array}{cc}
\sqrt{1}-\epsilon & 0 \\
0 & \sqrt{\epsilon}
\end{array}\right]
$$

There are several interesting properties of positive operators (28), such as: they are nonrepeatable (E2 is not equal to E), they are not unique, they are used when the basic elements in the Hilbert space of the model need not be orthogonal, they are used when there are more responses than there are basis states, this last property can be used in the decision-making models with noise in the system.

Quantum multiplayer games: as of now, we have mainly discussed some two player quantum games, and demonstrated that there are improvements from the classical counterparts. Benjamin et al. (2001) first introduced quantum multiplayer games, where there are $n$ players with $n>2$. In such games due to entanglement between choices of players, equilibria emerge (which in quantum theory language is called as pure coherent equilibrium) which de facto act like binding contracts, which prevent players from successfully betraying each other. Though the technicalities involved here is of higher degree, and it would not be possible to deal in details in this brief review, we can still spell out some basic features, as below, in two steps.

(1) Benjamin et al. (2001) quantize a simple multiplayer game (we can think of a Bit flipping game, where 0 and 1 bits are first sent to the players, who then can either do nothing or flip the bits sent to them, and then finally the output state is measured, hence as in the above penny flipping game, we can model non flipping by I and flipping by X GATE operations), by setting up a physical system. This is a crucial step since if some quantum physical features like entanglement has to be imbibed in the game, a physical set up is needed (for example in the PD game as we discussed above briefly); and

(2) Hence where as in the classical bit flipping game, each player has either to flip or not flip a bit sent to them, in quantum game case, they have Qbits rather than bits, and such Qbits are entangled. The game is designed in such a way that it is a generalization of the classical game. Or in other words when players restrict themselves from choosing from $\{\mathrm{I}, \mathrm{X}\}$ only the classical game payoff is recovered. A quantum strategy then can be $\mathrm{J}=1 / \sqrt{ } 2\left(I^{\otimes \mathrm{n}}+i X^{\otimes \mathrm{n}}\right)$, where $\mathrm{J}$ is an operator which commutes with any operator formed from I and $\mathrm{X}$ in the subspaces of different $\mathrm{Qbits}$.

\section{Concluding remarks}

This field of "quantum games" has emerged strongly since early 2000s, when Mathematicians started demonstrating that if "rational" agents share some physical systems (like quantum circuits or even very basic optical polarizers as we have shown) on which they can operate/measure some things, which can be mapped to their strategic moves, then there can arise superior Nash Equilibria.

The famous prisoners dilemma game is shown to generate superior NE (in one shot) if players share a physical system among them which demonstrate some special features like Entanglement. The main trick is to re-design the standard game in such a way that new strategies emerge, which are Pareto improvements.

However, there have been a lot of debates in the literature as to what bare min requirement generates such new strategies? It seems from different studies that pure quantum entanglement (or equivalently 'Bell inequality' violations) may just be a too strong condition, rather another weaker condition known as 'classical entanglement' may fit in.

We have demonstrated this recently in our "classical optical modelling" of PD game, but a general mathematical proof might be of fundamental value. 


\section{Notes}

1. CP stands here for Classical Probability Theory. QP for quantum probability.

2. CHSH inequalities were suggested [4] for testing any presence of non-local correlations in experimental outcomes, and is widely used in quantum theory framework.

3. GATES are basically logical operations, which take one or multiple Binary inputs and generates one output, widely used in classical and quantum information theory.

4. Poincare sphere is a widely used tool for describing states, like polarization states in Optics as well as in information theory in general. https://encyclopediaofmath.org/wiki/Poincar $\% \mathrm{C} 3 \%$ A9_sphere

5. https://en.wikipedia.org/wiki/Unitary_matrix

\section{References}

Basar, T. and Olsder, G.J. (1999), Dynamic Noncooperative Game Theory, SIAM Series in Classics in Applied Mathematics, Philadelphia, PA.

Benjamin, S.C. and Hayden, P.M. (2001), "Physical review A", Physical Review A, Vol. 64 No. 3, 64, 030301 N. F. Johnson, Phys. Rev. A 63, 020302.

Bruza, D., Wang, Z. and Busemeyer, J.R. (2015), "Quantum cognition: a new theoretical approach to psychology”, Trends in Cognitive Sciences, Vol. 19 No. 7, pp. 383-393.

Busemeyer, J.R. and Bruza, P. (2012), Quantum Models of Cognition and Decision Making, Cambridge University Press, Cambridge.

Clauser, J.F., Horne, M.A., Shimony, A. and Holt, R.A. (1969), "Proposed experiment to test local hiddenvariable theories", Physical Review Letters, Vol. 23 No. 15, p. 880.

Du, J., Xu, X., Li, H., Zhou, X. and Han, R. (2001), "Entanglement playing a dominating role in quantum games", Physics Letters A, Vol. 289 Nos 1/2, pp. 9-15.

Fehr, E. and Schmidt, K. (1999), “A theory of fairness, competition, and cooperation”, The Quarterly Journal of Economics, Vol. 114 No. 3, pp. 817-868.

Fine, A. (1982), "Joint distributions, quantum correlations, and commuting observables", Journal of Mathematical Physics, Vol. 23 No. 7, p. 1306.

Fudenberg, D. and Levine, D. (1998), The Theory of Learning in Games, MIT Press, Cambridge, MA.

Ghose, P. and Mulherjee, A. (2014), "Entanglement in classical optics", Reviews in Theoretical Science, Vol. 2 No. 4.

Haven, E. and Khrennikov, A. (2013), Quantum Social Science, Cambridge University Press.

Haven, E. and Khrennikova, P. (2018), “A Quantum-Probabilistic paradigm: non-consequential reasoning and state dependence in investment choice", Journal of Mathematical Economics, Vol. 78 .

Iqbal, A. and Abbott, D. (2009), "Non-factorizable joint probabilities and evolutionarily stable strategies in the quantum prisoner's dilemma game", Physics Letters A, Vol. 373 No. 30, pp. 2537-2541.

Iqbal, A. and Abbott, D. (2010), "Constructing quantum games from a system of bell's inequalities", Physics Letters A, Vol. 374 Nos 31/32.

Kahneman, D., Slovic, P. and Tversky, A. (1982), Judgment under Uncertainty: Heuristics and Biases, Cambridge University Press, New York, NY.

Khrennikov, A.Y. (2015), “Quantum version of Aumann's approach to common knowledge: sufficient conditions of impossibility to agree on disagree", Journal of Mathematical Economics, Vol. 60, pp. 89-104. 
AJEB

4,3

Krueger, J.I., DiDonato, T.E. and Freestone, D. (2012), "Social projection can solve social dilemmas", Corrigendum. Psychological Inquiry, Vol. 23 No. 3, p. 315.

Li, Y., Qin, G., Zhou, X.Y. and Du, J.F. (2006), "The application of asymmetric entangled states in quantum games", Physics Letters A, Vol. 355 No. 6, pp. 447-451.

Luders, G. (1951), Annalen Der Physik, Vol. 322 No. 8, available at: https://doi.org/10.1002/andp.200610207

Morgenstern, O. (1976), "The collaboration between Oskar Morgenstern and John von Neumann on the theory of games", Journal of Economic Literature, Vol. 14, pp. 805-808.

Myerson, R.B. (2013), Game Theory, Harvard University Press.

Rasmusen, E. (1994), Games and Information, 2nd ed., Oxford University Press, Cambridge, MA.

Shafir, E. and Tversky, A. (1992), "Thinking through uncertainty: nonconsequential reasoning and choice", Cognitive Psychology, Vol. 24 No. 4, pp. 449-474.

Tversky, A. and Kahneman, D. (1973), “Availability: a heuristic for judging frequency and probability”, Cognitive Psychology, Vol. 5 No. 2, pp. 207-232.

Yearsley, J.M. (2017), “Advanced tools and concepts for quantum cognition: a tutorial”, Journal of Mathematical Psychology, Vol. 78, pp. 24-39.

\section{Further reading}

Feynman, R., Leighton, R.B. and Sands, M. (2020), Feynman Lectures on Physics is a Very Widely Used Source, Vol. 3.

Khrennikov, A. (2010), Ubiquitous Quantum Structure: From Psychology to Finance, Springer.

Patra, S. and Ghose, P. (2020), "Preprint", available at: www.researchgatenet/publication/ 341575648_Classical_Optical_Modelling_of_the_'Prisoner's_Dilemma'_Game

\section{Appendix}

Basics of QP(Quantum Probability) framework

We begin with a brief comparison between classical probability theory (CPT) and quantum probability theory (QPT):

The main features of CPT are:

- Events are represented by sets, which are subsets of. Sample space, sigma algebra, measure (probability)*, are the main features of the related Kolmogorov measure theory.

- Boolean logic is the type of compatible logic with CPT, which allows for deductive logic, and basic operations like union and intersection of sets, DeMorgan Laws of set theory are valid.

- Conditional probability: $\mathrm{P}(\mathrm{a} \mid \mathrm{b})=\mathrm{p}(\mathrm{a}$ and $\mathrm{b}) / \mathrm{p}(\mathrm{b}) ; \mathrm{p}(\mathrm{b})>0$ We see conditional probability is a direct consequence of Boolean operations

The main features of Quantum Probability Theory are:

State space is a complex linear normed vector space: Hilbert space; Finite/infinite D, symbolized as $\mathrm{HH}$ is endowed with a scalar product (positive definite), norm, and an orthonormal basis, nondegenerate Any state can be visualized as a ray in this space Superposition principle: which states that a general state can be written as a linear superposition of 'basis states', in information theory language the basis states are $\mid 0>$ or $\mid 1>$.

Measurement: most of the times projection postulate; Measurement implies projection onto a specific Eigen sub-space. Probability, updating can be visualized as sequential projections on Eigen subspaces Non -Boolean logic is compatible with such state space structure, which means violation of commutation and associative properties. 


\section{The main features of Non-Boolean logic are}

Algebra of events is prescribed by quantum logic. Events form an event ring R, possessing two binary operations, addition and conjunction $\mathrm{P}(\mathrm{A} \mathrm{U} B)=\mathrm{P}(\mathrm{BUA})$ (this Boolean logic feature is invariant in Quantum logic). $\mathrm{P}\{\mathrm{AU}(\mathrm{BUC})\}=\mathrm{P}\{(\mathrm{AUB}) \mathrm{U}(\mathrm{AUC})\}$ (associative, property also holds good):

$$
\mathrm{AUA}=\mathrm{A}(\text { idempotency) }
$$

$\mathrm{P}(\mathrm{A}$ and $\mathrm{B})$ \# $\mathrm{P}(\mathrm{B}$ and $\mathrm{A}$ ) (non -commutativity, incompatible variables) $\mathrm{A}$ and (B UC) \# (A and B) U (A and C) (no distributivity)

The fact that distributivity is absent in quantum logic was emphasized by Birkhoff and von Neumann. Suppose there are two events B1 and B2 that, when combined, form unity, B1 $\cup$ B2 = 1. Moreover, $\mathrm{B} 1$ and $\mathrm{B} 2$ are such that each of them is orthogonal to a nontrivial event $\mathrm{A} \# 0$, hence $\mathrm{A} \cap \mathrm{B} 1=\mathrm{A} \cap \mathrm{B} 2=0$. According to this definition, $\mathrm{A} \cap(\mathrm{B} 1 \cup \mathrm{B} 2)=\mathrm{A} \cap 1=\mathrm{A}$. But if the property of distributivity were true, then one would get $(\mathrm{A} \cap \mathrm{B} 1) \cup(\mathrm{A} \cap \mathrm{B} 2)=0$. This implies that $\mathrm{A}=0$, which contradicts the assumption that $\mathrm{A} \# 0$.

\section{The main features of quantum-like modeling of belief states are}

Bruza et al. (27): cognitive modelling based on quantum probabilistic frame work, where the main objective is assigning probabilities to events space of belief is a finite dimensional Hilbert space $\mathrm{H}$, which is spanned by an appropriate set of basis vectors observables are represented by operators (positive operators/Hermitian operators) which need not commute:

$$
[\mathrm{A}, \mathrm{B}]=\mathrm{AB}-\mathrm{BA}=0
$$

Generally, any initial belief state is represented by density matrix/operator, outer product of $\psi$ with itself $\rho=|\psi\rangle\langle\psi|$, this is a more effective representation since it captures the ensemble of beliefs:

Pure states and mixed states

Mixed states: $\sum \mathrm{w}|\psi\rangle\langle\psi|$, hence mixed state is an ensemble of pure states with w's as probability weights.

Some properties of $\rho: \rho=\left(\rho^{*}\right)$ T, for pure states $\rho=\rho^{\wedge} 2$, where T stands for transpose operation. $(\psi, \rho \psi)>0$ : positivity, Trace $\rho=1$.

Measuring the probability of choosing one of the given alternatives, which is represented by the action of an operator on the initial belief state.

While making decision superposition state collapses to one single state (can be captured by the Eigen value equation).

Observables in QPT represented by Hermitian operators:

$$
\mathrm{A}=\left(\mathrm{A}^{*}\right) \wedge \mathrm{T}
$$

$\mathrm{E}(\mathrm{A})=\operatorname{Tr}(\mathrm{A} \rho)$, every time measurement is done one of the Eigenvalues of the $\mathrm{A}$ is realized.

$A=\sum \mathrm{aP}$ spectral decomposition rule: a's are the Eigen values and P's are the respective projectors which projects the initial state to the Eigen subspace with a specific a:

$$
\text { Trace formula : } \mathrm{p}(\mathrm{ai})=\operatorname{Tr}(\operatorname{Pi} \rho)
$$

Quantum-like modelling in game theory 
AJEB

4,3

As soon as the measurement is done the state $\rho^{\prime}: \mathrm{Pi} \rho \mathrm{Pi} / \operatorname{Tr}(\mathrm{Pi} \rho)$ Simultaneously updating of the agents' belief state.

A quick review of formula for total probability/law of total probability (Ltp), modified in quantum-likeset up.

First, we see the LTP in classical set theory as below:

$$
\mathrm{P}(\operatorname{Band}(\text { AorC }))=\mathrm{P}(\text { BandA })+\mathrm{P}(\text { BandC })(\text { measuretheoreticadditivity })
$$

$$
\mathrm{P}(\text { Band } \mathrm{A})=\mathrm{P}(\mathrm{A}) \mathrm{P}(\mathrm{B} \mid \mathrm{A}) \text {, and }, \mathrm{P}(\mathrm{B} \mid \mathrm{A})=\mathrm{P}(\operatorname{Band} \mathrm{A}) / \mathrm{P}(\mathrm{A})
$$

Hence, it follows:

$$
\mathrm{P}(\mathrm{B} \mid(\mathrm{A} \text { or } \mathrm{C}))=\mathrm{P}(\mathrm{B} \mid \mathrm{P}(\mathrm{A} \text { or } \mathrm{C})=\{\mathrm{P}(\mathrm{B} \mid \mathrm{A}) * \mathrm{P}(\mathrm{A})+\mathrm{P}(\mathrm{B} \mid \mathrm{C}) * \mathrm{P}(\mathrm{C})\} / \mathrm{P}(\mathrm{A} \text { or } \mathrm{C})
$$

Hence, in particular if $\mathrm{P}(\mathrm{A}$ or $\mathrm{C})=1$, then $\mathrm{P}(\mathrm{B})=\left\{\mathrm{P}(\mathrm{B} \mid \mathrm{A})^{*} \mathrm{P}(\mathrm{A})+\mathrm{P}(\mathrm{B} \mid \mathrm{C}) * \mathrm{P}(\mathrm{C})\right\}$, this is the LTP (law of total probability) as we know in familiar CPT.

But in the QPT (quantum probability theory) additivity does not follow, which means LTP is violated since there are interference terms

To get the modified LTP as in non Kolgomorovian QDT set up we have to go through the concept of positive valued operators (POVM) as below:

A positive operator valued measure (POVM) is a family of positive operators $\{\mathrm{Mj}\}$ such that $\sum \mathrm{Mj}=\mathrm{I}$, where I is the unit operator. It is convenient to use the following representation of POVMs:

$$
\mathrm{Mj}=\mathrm{V} * \mathrm{j} \mathrm{Vj}
$$

where $\mathrm{Vj}: \mathrm{H} \rightarrow \mathrm{H}$ are linear operators. A POVM can be considered as a random observable. Take any set of labels $\alpha 1, \ldots, \alpha \mathrm{m}$, e.g. for $\mathrm{m}=2, \alpha 1=$ yes, $\alpha 2=$ no. Then the corresponding observable takes these values (for systems in the state $\rho$ ) with the probabilities $\mathrm{p}(\alpha \mathrm{j}) \equiv \mathrm{p} \rho(\alpha \mathrm{j})=\operatorname{Tr} \rho \mathrm{Mj}=$ $\operatorname{TrVj} \rho \mathrm{V}^{*} \mathrm{j}$.We are also interested in the post-measurement states. Let the state $\rho$ was given, a generalized observable was measured and the value $\alpha \mathrm{j}$ was obtained. Then the output state after this measurement has the form: $\rho \mathrm{j}=\mathrm{Vj} \rho \mathrm{V} * \mathrm{j} /(\operatorname{Tr} \mathrm{Vj} \rho \mathrm{V} * \mathrm{j})$.

Both order effects and interference terms in LTP can be demonstrated using POVMConsider two generalized observables a and b corresponding to POVMs $\mathrm{Ma}=\{\mathrm{V} * \mathrm{j} \mathrm{Vj}\}$ and $\mathrm{Mb}=\{\mathrm{W} * \mathrm{j} \mathrm{Wj}\}$, where $\mathrm{Vj} \equiv \mathrm{V}(\alpha \mathrm{j})$ and $\mathrm{Wj}=\mathrm{W}(\beta \mathrm{j})$ correspond to the values $\alpha \mathrm{j}$ and $\beta \mathrm{j}$. If there is given the state $\rho$ the probabilities of observations of values $\alpha \mathrm{j}$ and $\beta \mathrm{j}$ have the form:

$$
\operatorname{pa}(\alpha)=\operatorname{Tr} \rho \mathrm{Ma}(\alpha)=\operatorname{TrV}(\alpha) \rho \mathrm{V}^{*}(\alpha), \mathrm{p}(\beta)=\operatorname{Tr} \rho \mathrm{Mb}(\beta)=\operatorname{Tr} \mathrm{W}(\beta) \rho \mathrm{W}^{*}(\beta) .
$$

Now we consider two consecutive measurements: first the a-measurement and then the bmeasurement. If in the first measurement the value $\mathrm{a}=\alpha$ was obtained, then the initial state $\rho$ was transformed into the state:

$$
\rho \mathrm{a}(\alpha)=\mathrm{V}(\alpha) \rho \mathrm{V}^{*}(\alpha) /\left(\operatorname{TrV}(\alpha) \rho \mathrm{V}^{*}(\alpha)\right)
$$

For the consecutive b-measurement, the probability to obtain the value $\mathrm{b}=\beta$ is given by $(\beta \mid \alpha)=\operatorname{Tr} \rho \mathrm{a}(\alpha) \mathrm{Mb}(\beta)=\operatorname{Tr} \mathrm{W}(\beta) \mathrm{V}(\alpha) \rho \mathrm{V}^{*}(\alpha) \mathrm{W}^{*}(\beta) /\left(\operatorname{Tr} \mathrm{V}(\alpha) \rho \mathrm{V}^{*}(\alpha)\right)$ 
This is the conditional probability to obtain the result $\mathrm{b}=\beta$ under the condition of the result $\mathrm{a}=\alpha$. We set $\mathrm{p}(\alpha, \beta)=\mathrm{pa}(\alpha) \mathrm{p}(\beta \mid \alpha)$.

Now since operators need not commute $\mathrm{p}(\alpha, \beta)=\mathrm{p}(\beta, \alpha)$.

We recall that, for two classical random variables a and b which can be represented in the Kolmogorov measure-theoretic approach, the formula of total probability (FTP) has the form $\mathrm{pb}(\beta)=$ $\sum \operatorname{pa}(\alpha) \mathrm{p}(\beta \mid \alpha)$.

Further we restrict our consideration to the case of dichotomous variables, $\alpha=\alpha 1, \alpha 2$ and $\beta=$ $\beta 1, \beta 2$.

FTP with the interference term for in general non-pure states given by density operators and generalized quantum observables given by two (dichotomous) PVOMs:

$$
\operatorname{pb}(\beta)=\operatorname{pa}(\alpha 1) \mathrm{p}(\beta \mid \alpha 1)+\operatorname{pa}(\alpha 2) \mathrm{p}(\beta \mid \alpha 2)+2 \lambda \sqrt{ }\{\operatorname{pa}(\alpha 1) \mathrm{p}(\beta \mid \alpha 1) \operatorname{pa}(\alpha 2) \mathrm{p}(\beta \mid \alpha 2)\},
$$

or by using ordered joint probabilities $\mathrm{pb}(\beta)=\mathrm{p}(\alpha 1, \beta)+\mathrm{p}(\alpha 2, \beta)+2 \lambda \beta \sqrt{ } \mathrm{p}(\alpha 1, \beta) \mathrm{p}(\alpha 2, \beta)$. Here the coefficient of interference $\lambda$ has the form: $\lambda=\operatorname{Tr} \rho\left\{\mathrm{W}^{*}(\beta) \mathrm{V}^{*}(\alpha \mathrm{i}) \mathrm{V}(\alpha \mathrm{i}) \mathrm{W}(\beta)-\mathrm{V}^{*}(\alpha \mathrm{i})\right.$ $\left.\mathrm{W}^{*}(\beta) \mathrm{W}(\beta) \mathrm{V}(\alpha \mathrm{i})\right\} / 2 \sqrt{ } \mathrm{pa}(\alpha 1) \mathrm{p}(\beta \mid \alpha 1) \mathrm{pa}(\alpha 2) \mathrm{p}(\beta \mid \alpha 2)$ Introduce the parameters:

$$
\begin{aligned}
\gamma \alpha \beta & =\operatorname{Tr} \rho \mathrm{W}^{*}(\beta) \mathrm{V}^{*}(\alpha) \mathrm{V}(\alpha) \mathrm{W}(\beta) /\left(\operatorname{Tr} \rho \mathrm{V}^{*}(\alpha) \mathrm{W}^{*}(\beta) \mathrm{W}(\beta) \mathrm{V}(\alpha)\right) \\
& =\mathrm{p}(\beta, \alpha) / \mathrm{p}(\alpha, \beta)
\end{aligned}
$$

This parameter is equal to the ratio of the ordered joint probabilities of the same outcome, but in the different order, namely, "b then a" or "a then b". Then:

$$
\begin{aligned}
\text { Interferenceterm } \lambda= & 1 / 2\left\{\sqrt { } \left(\mathrm{p}(\alpha 1, \beta) / \mathrm{p}(\alpha 2, \beta)^{*}(\gamma \alpha 1 \beta-1)\right.\right. \\
& +\sqrt{ }\left(\mathrm{p}(\alpha 2, \beta) / \mathrm{p}(\alpha 1, \beta)^{*}(\gamma \alpha 2 \beta-1)\right.
\end{aligned}
$$

In principle, this coefficient can be larger than one. Hence, it cannot be represented as $\lambda=\cos \theta$ for some angle ("phase") $\theta$, cf. However, if POVMs Ma and Mb are, in fact, spectral decompositions of Hermitian operators, then the coefficients of interference are always less than one, i.e. one can find phases $\theta$.

One important note is that such phase terms cannot always be expressed in trigonometric terms, Hyperbolic phase terms are also possible, which are typical of results obtained from decision-making models (Haven and Khrennikov, op cit).

\section{Entanglement mathematics}

As we have seen throughout that quantum theory allows superposition of the basis states to form new states, many of such superpositions, but not all, poses the quality of entangled states. For example, we start with a qubit system (i.e. a system which has only two basis states $\mid 0>$ and $\mid 1>$, where they may represent up and down states, for example in decision-making models they represent belief sets of decision makers as up state or down state related to any future event), now such a system can be written in superpositions of the basis states in a number of ways:

$\mid x>=1 / \sqrt{ } 2\{|00>+| 11>\}$, this state can be called as an entangled state, since say if these qubits are given to Alice and Bob, and even they are separated light years apart, if Alice measures her system there is always a 50-50 chance of finding a $\mid 0>$ or $\mid 1>$, however as soon as she discovers that it is determined with $100 \%$ probability that Bob has to have $\mid 0>$ in the first case and $\mid 1>$ in the second case. 
AJEB

4,3

66

Hence, there is no superluminal communication happening, only that subsystems are in a random state and the system as a whole is in a pure state.

Again, another hallmark of such states is that mathematically they are not separable, in the sense that $\mid x>$ cannot be written as a sum over tensor products of only $\mid 0>$ or $\mid 1>$.

Comparatively, separable states are like $\mid \mathrm{y}>=1 / \sqrt{ } 2\{|00>+| 01>\}$, in such a case Alice will always with probability 1 measure her subsystem to be in $|0\rangle$ but Bob still will have a $50 \%$ chance of $\mid 1>$ or $\mid 0>$, again $\mid \mathrm{y}>$ can be separated as $1 / \sqrt{ } 2\{\mid 0>(|0>+| 1>)\}$ which means a tensor product between $\mid 0>$ and the superposition of $\mid 0>$ and $\mid 1>$.

Measure of degree of entanglement: concurrence measure is a type of measure of degree of entanglement, say a general entangled state is written as: $\mathrm{a}|00>+\mathrm{b}| 01>+\mathrm{c}|10>+\mathrm{d}| 11>$

Then the state is maximally entangled if $|\mathrm{ad}-\mathrm{bc}|=1$, and there is no entanglement if $|\mathrm{ad}-\mathrm{bc}|=0$.

\section{Corresponding author}

Sudip Patra can be contacted at: spatra@jgu.edu.in

For instructions on how to order reprints of this article, please visit our website: www.emeraldgrouppublishing.com/licensing/reprints.htm Or contact us for further details: permissions@emeraldinsight.com 